\title{
FLAME ACCELERATION AND OVERPRESSURE DEVELOPMENT IN A SEMIOPEN TUBE WITH REPEATED OBSTACLES
}

\author{
L. X. YU, W. C. SUN AND C. K. WU \\ Institute of Mechanics \\ Chinese Academy of Sciences \\ Beijing 100080, P. R. China
}

\begin{abstract}
An investigation of the dependence of turbulent flame acceleration and overpressure in a semiopen tube on the configuration of obstacles has been performed in a flame propagation tube with one end closed and the other open, having an $80 \mathrm{~mm}$ inner diameter, a $5 \mathrm{~m}$ length, and repeated obstacles. Three kinds of obstacle shapes have been used for various blockage ratios and spacing of obstacles. There is evidence that the influence of the obstruction characteristics on the flame speed and overpressure is different in the various flame regimes. In the low-speed combustion regime, the highest terminal flame speed is obtained with the blockage ratio of $\mathrm{BR}=0.3-0.4$. In the choking regime, the maximum flame speed is insensitive to the blockage ratio, and a blockage ratio of about 0.5 generates the highest peak overpressure. In the detonation regime, the maximum flame speed and overpressure decrease with increasing blockage ratio due to the severe momentum losses induced by the blockage effect of the obstacles, and at the same time, the detonative range is observed to become narrower. The steady flame speed is independent of the shape of obstacles when the blockage ratio is the same, and the spacing of obstacles plays a role only in determining the flame acceleration rate rather than the steady flame speed; the highest mean flame speed and peak overpressure are obtained when the spacing of obstacles is about equal to the inner diameter of the flame tube. In addition, the unsteady compressible flow model with correction for the influence of turbulence Mach number on viscosity dissipation and pressure dilatation was formulated, and numerical studies using the eddy-break-up combustion model were made to predict the flame acceleration and the development of overpressure in the tube. The comparison of calculated overpressure and flame speeds with experimental data shows good agreement.
\end{abstract}

\section{Introduction}

In most cases, a freely propagating flame is intrinsically unstable because the combustion process and the flow ahead are intimately coupled: the propagation of a flame will induce flow in the unburned gas. If repeated obstacles are positioned along the path of flame propagation, this flow will result in the production of turbulence. The turbulence will increase the burning velocity by distorting the flame front, the stretched flame will enhance the turbulence, and so on. Owing to this positive feedback mechanism between the burning rate of the flame and gas-dynamic flow structure and to the presence of wall friction and heat release, the flame continuously accelerates and either transits from deflagration to detonation or eventually reaches a final quasisteady state, and the maximum flame speed is obtained.

In past decades, the problem of flame acceleration due to obstacle-produced turbulence has been studied by various authors. These studies can be classified into two categories:

1. Flame propagation in closed tubes [1-3]. The influence of obstacles on flame acceleration has been investigated in various kinds of tubes and vessels, and some meaningful results were obtained in the theory and the numerical simulation. For example, turbulent flame acceleration in closed tubes has been studied by Lee et al. [46]; their results demonstrated that the obstacle had a dramatic influence on flame propagation. For sensitive mixtures such as $\mathrm{H}_{2}$ /air or $\mathrm{C}_{2} \mathrm{H}_{2}$ / air, there exist four distinct regimes: the quenching regime, the choking regime, the qusai-detonation regime, and the detonation regime.

2. Flame propagation in vented tubes. Some experimental and theoretical work in open tubes has been done by Moen [7], Hjertager [8], Chan [9], Bradley $[10,11]$, and others. However, compared to work on closed tubes, there is much less experimental data and theorical analysis of flame propagation in open tubes, and most experiments have been done in large-scale tubes or vessels.

There are also practical applications of explosions in vented vessels. For instance, a new type of ashcleaning facility now widely used in power plants in China is a semiopen system burning gaseous fuels [12]. Therefore, from a practical point of view, it is 


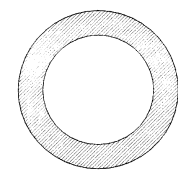

a) obstacle $\mathrm{A}$

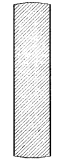

b) obstacle $B$

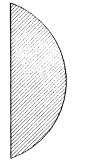

c) obstacle C
FIG. 1. Schematic diagram of obstacle shapes.

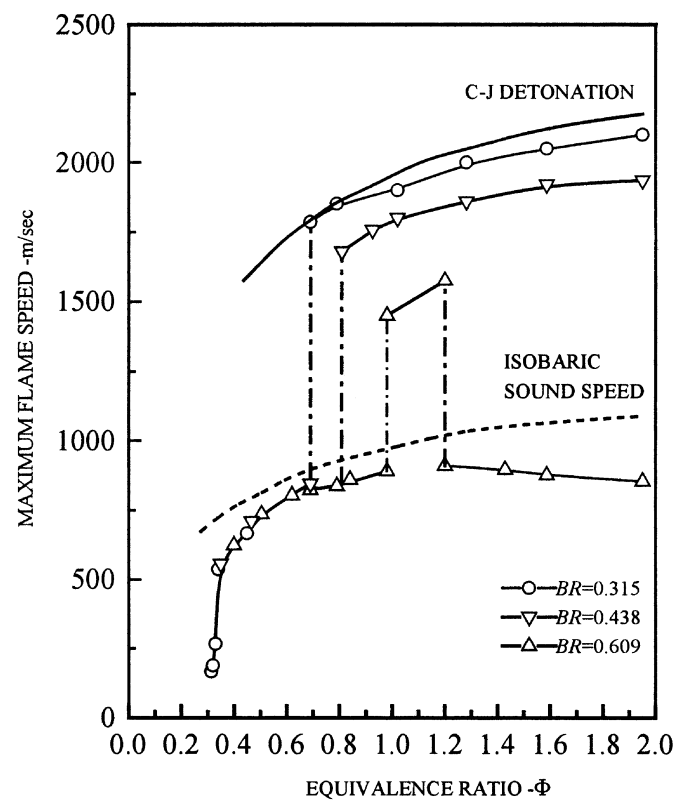

FIG. 2. Maximum flame speed for hydrogen/air mixtures with various blockage ratios, obstacle A.

also important to explore the mechanism of unsteady flame acceleration in semiopen tubes. An investigation of the dependence of turbulent flame acceleration and overpressure in the tube on the configuration of obstacles has been carried out with various fuels by changing the obstacle shape, spacing, and blockage ratio, and a numerical study of the development of flame speeds and transient overpressure was performed with an unsteady compressible flow model, the predicted values being compared with measured data. The present paper reports some of the results obtained in an attempt to enrich the study of and deepen the understanding of turbulent flame propagation in partially obstructed semiopen tubes.

\section{Experimental Details}

The flame tube used was a semiopen obstructed tube of $80 \mathrm{~mm}$ inner diameter and $5 \mathrm{~m}$ length. The blockage ratios were $0.212,0.315,0.438,0.609$, and 0.75 . The blockage ratio is defined as $\mathrm{BR}=$ blocking area of obstruction/cross-section area of flame tube.
In order to compare the flame speeds and overpressures caused by different obstacle configurations, three shapes of obstacle were used in the experiment; as shown in Fig. 1, all three obstacles were thin plates. The obstacles were all arranged in periodical succession a distance $W$ apart.

When the tube was fully filled with a premixed gas mixture, the inlet valve was closed, and ignition was initiated by an electrical spark; then, the initial flame propagated along the flame tube. The flame accelerated through the tube from the closed to the open end due to the turbulence produced. In order to monitor the development of flame speeds and overpressure, ionization gap probes (on the centerline) and pressure transducers (on the wall) were located at different positions along the tube. The signals were recorded on an eight-channel high-speed digital recorder with a top sampling frequency of $400 \mathrm{kHz}$, so that the flame speeds and transient pressure in the tube could be measured.

\section{Results and Discussion}

The comparison of the steady-state flame speeds for three blockage ratios of obstacle A for various equivalence ratios of hydrogen/air mixture is given in Fig. 2. It should be pointed out that the flame speed as measured here is the sum of the burning velocity and the displacement velocity of the gas ahead of the flame. The flame propagation regimes transit from the low-speed combustion regime to the choking regime, and then to the detonation regime; two corresponding velocity jumps in the flame speed can be clearly observed. In the detonation regime, the flame speeds decrease with the blockage ratio due to the increase in pressure losses across the obstacles; for example, the detonation speeds are 1900, 1800 , and $1480 \mathrm{~m} / \mathrm{s}$, respectively, corresponding to blockage ratios of $0.315,0.438$, and 0.609 . At the same time, the detonative range is observed to become narrower with increasing blockage ratio. This suggests that there exists a critical blockage ratio beyond which the transition to detonation would not be possible for a hydrogen/air mixture of any given equivalence ratio.

Figure 3 shows the comparison of flame speeds in water gas/air mixture for three different shapes of obstacle. The flame speed profiles for various conditions are more or less the same for the same blockage ratio and spacing $W$ of obstacles. Therefore, for the shapes $\mathrm{A}, \mathrm{B}$, and $\mathrm{C}$, the flame acceleration rate is independent of the shape of obstacle.

When a flame propagates along a tube with repeated obstacles, flame propagation can be considered as a sequence of combustion events in the chambers between the blockage plates and flow across the plates. In each chamber, the wall is smooth so that it is reasonable to assume that the 


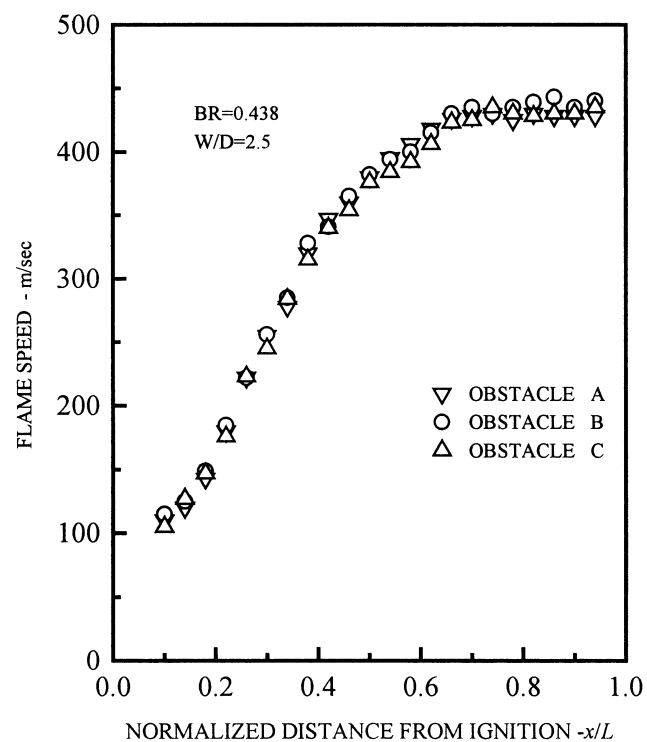

FIG. 3. Variation of flame speed along the flame tube for the various shapes of obstacles $(\mathrm{BR}=0.438, W / D=2.5)$.

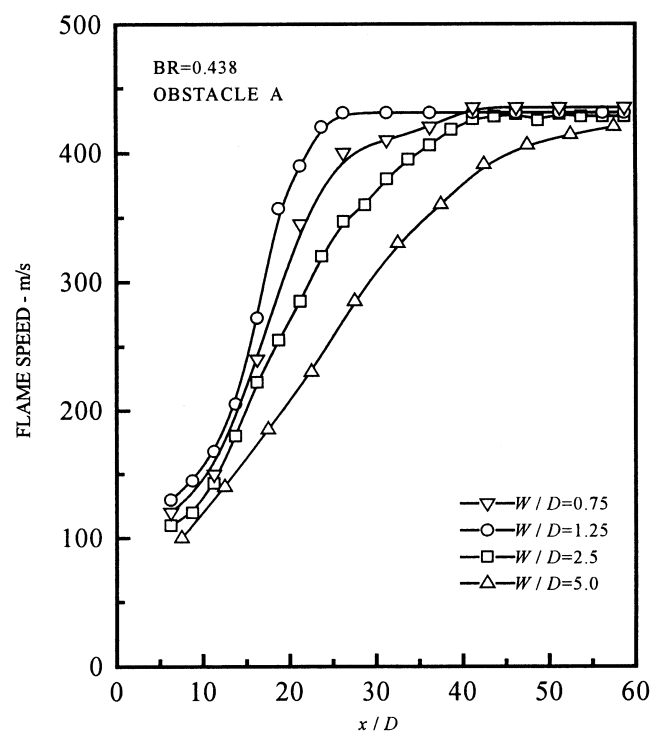

FIG. 4. Variation of flame speed of water gas mixture along the flame tube for various spacing of obstacles $(\mathrm{BR}=$ 0.438 , obstacle A).

flame propagates at a more or less uniform velocity (relative to the acceleration across the obstacles). Only in the outlet of the chamber does large flame distortion exist, resulting in rapid flame acceleration. It is evident that the speeds of premixed flames in the outlet of the chamber depend mainly on the blockage area of the obstacle rather than the chamber length. Thus, one may expect that, in the case

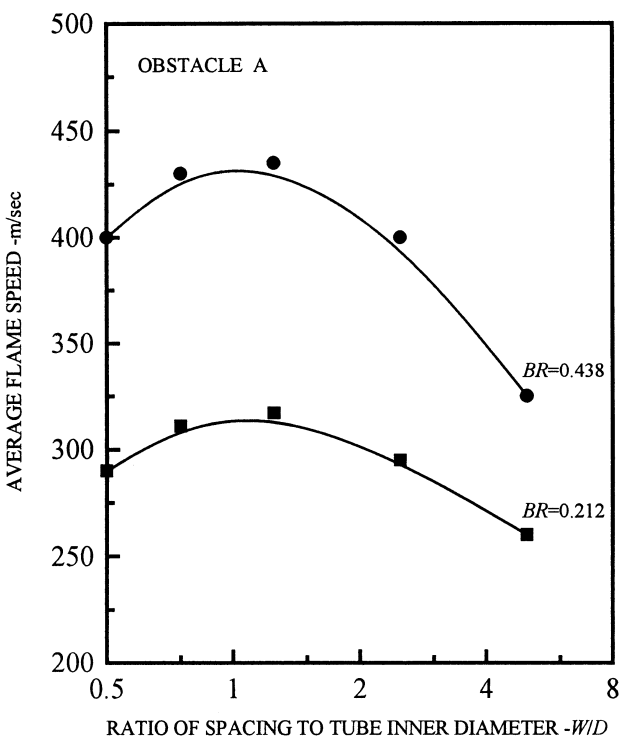

FIG. 5. Variation of mean flame speed in water gas/air mixture for various spacing of obstacles at $\mathrm{BR}=0.438$ and 0.212 .

of the same blockage ratio, the terminal qusai-steady flame speed would depend on the number of obstacles if the flame tube is long enough to make the flame propagation reach a steady state. Fig. 4 shows the variation of flame speeds in water gas/air for various obstacle spacings. It can be concluded that the spacing of obstacles plays a role only in determining the flame acceleration rate rather than the final flame speed. It can be seen from Fig. 5 that the highest average flame speed in the water gas/air mixture is obtained when the obstacle spacing $W$ is about equal to the inner diameter of flame tube $D$, that is, $W / D \approx 1$, for blockage ratios of both 0.438 and 0.212 . This means that the distance at which flame propagation reaches the terminal steady state is shortest, which is somewhat the same as the result of $W / D=0.8$ reported by Hjertager [8].

The peak overpressure variation with equivalence ratio of hydrogen/air mixture is plotted in Fig. 6 for various blockage ratios. In the low-speed combustion regime, the peak pressure is below the theoretical constant volume explosion pressure. The rapid increase in pressure is found as the combustion transits to the choking regime. In the whole choking regime, the peak overpressure is somewhat above the constant volume explosion pressure. In the range of equilvance ratios where the transition from deflagration to detonation occurs, the pressure shows only a moderate increase, which is about midway between the equilibrium Chapman-Jouget (C-J) detonation pressure and the constant volume explosion pressure, although a distinct jump in flame speed is observed in Fig. 2. This can be attributed to two 


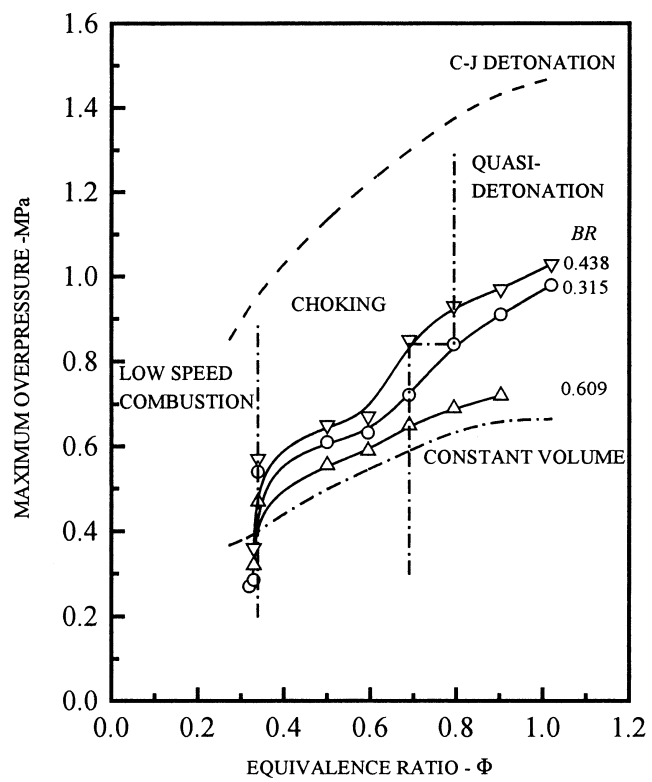

FIG. 6. Variation of the peak overpressure with equivalence ratio of hydrogen/air mixtures for various blockage ratios with obstacle $\mathrm{A}$.

aspects: (1) The pressure tranducers are positioned on the wall, while the ionization probes are located on the centerline. The flow and detonation wave structure in the tube with obstructions are not one dimensional. There are oblique waves and regions

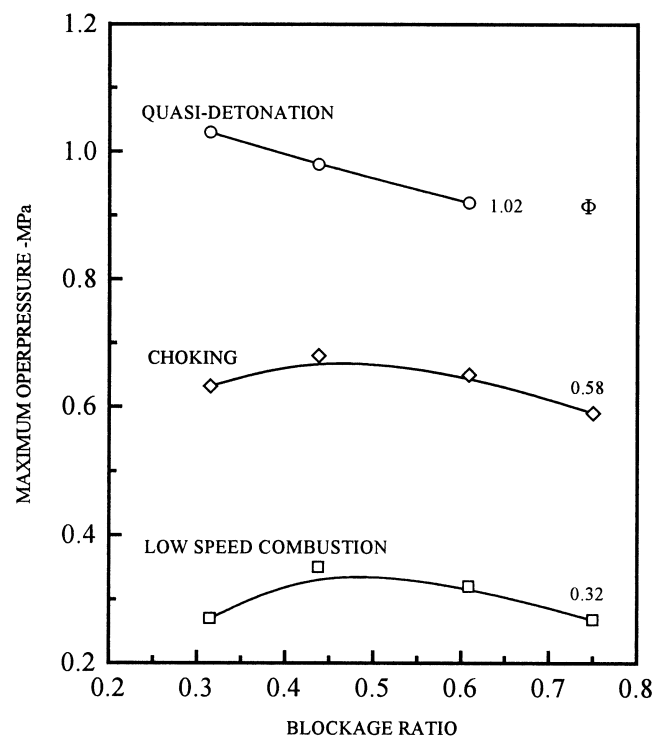

FIG. 7. Variation of maximum overpressure in hydrogen/ air mixtures with various blockage ratios of obstacle A in different flame propagation regimes.

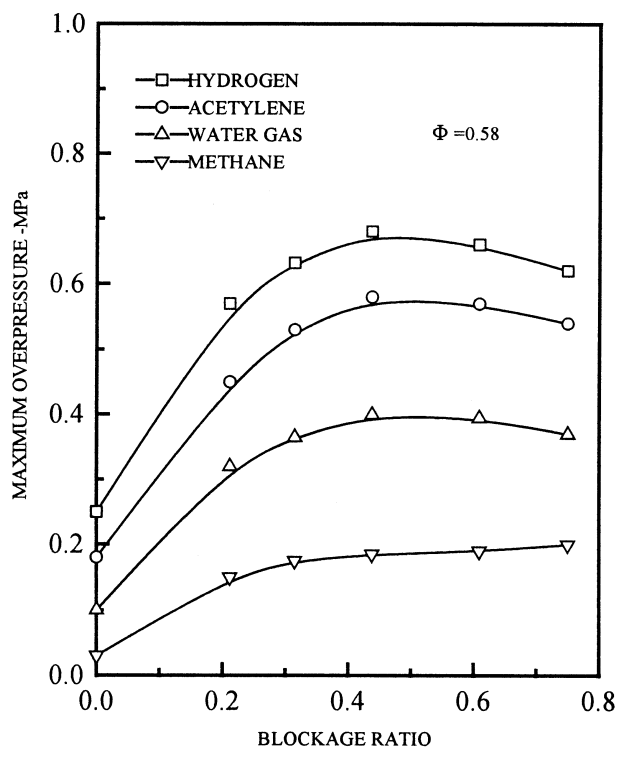

FIG. 8. Variation of peak pressure in the tube with various blockage ratios for four different kinds of fuel.

of lower velocities near the wall, which make the measured values smaller than the pressure behind the wave at the center. (2) There is non-synchronization in reaching the steady-state values between the propagation speed of the detonation wave and physical quantities behind the wave. It was pointed out [13] that the detonation pressure behind the wave only reached $75 \%$ of the C-J value when the detonation flame speed reached $99 \%$ of the normal C-J value, the detonation pressure would reach the normal C-J value after the detonation wave propagated past a considerably long distance, this is referred to as the "detonation increase process." The experiments of Craig [13] indicated that the pressure behind the detonation wave increased with the propagation distance, the detonation pressures went up $25 \%$, while the flame speeds rose only $1 \%$.

The dependence of pressure on the blockage ratio is not monotonic. In the low-speed combustion regime and the choking regime, the maximum overpressure increases with increasing blockage ratio when the blockage ratio is smaller than 0.5. However, the trend is reversed when the blockage ratio is larger than 0.5, as shown in Fig. 7. Nevertheless, in the detonation regime, the peak overpressure decreases with an increase in blockage ratio due to the severe pressure differences across the obstacle.

The variation of peak pressure in the tube with various blockage ratios for four different kinds of fuel is shown in Fig. 8. It is noted that $\mathrm{BR}=0$ represents the tube without obstacles. It is observed that the obstacles have a dramatic influence on the development of the pressure in the tube. In the tube 


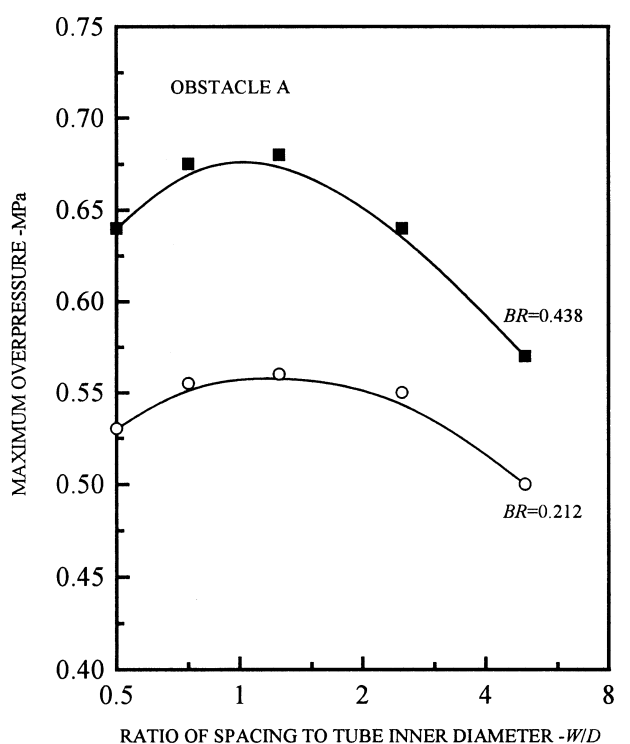

FIG. 9. Variation of maximum overpressure in hydrogen/ air mixtures with various spacings of obstacle at two different blockage ratios of obstacle A.

without obstacles, at an equivalence ratio of 0.58 , the maximum overpressures for methane, water gas, acetylene, and hydrogen are $0.03,0.1,0.18$, and $0.25 \mathrm{MPa}$, respectively. With obstacles in the flame tube, even a relatively small blockage leads to a considerable increment in the overpressure in the tube, the corresponding overpressures for the above four gases with a blockage ratio of 0.212 go up to 0.15 , $0.32,0.45$, and $0.57 \mathrm{MPa}$, respectively, increasing by $500 \%, 320 \%, 250 \%$, and $228 \%$, respectively.

The maximum overpressure of a hydrogen/air mixture in the tube as function of obstacle spacing is shown in Fig. 9. Strong variation with obstacle spacing can be observed. The peak pressure in the tube first increases and then decreases with increasing obstacle spacing, the highest peak pressure is obtained for an obstacle spacing equal to the inner diameter of the flame tube, which is the same as the trend for flame speed.

\section{Numerical Simulation}

It can be seen that for the more sensitive fuels under certain conditions of equivalence ratio and obstacle configuration, transition to detonation occurs. When this happens, the combustion wave is accompanied by a shock wave in the tube. The propagation mechanism of a detonation is so different from that of a deflagration that they can not be studied from a unified theory. The effects of shock waves on combustion processes are not considered in the following numerical study, and prediction is limited to the de- velopment of a subsonic deflagration. Owing to the high Mach number of the turbulent flow in the flame propagation, the influence of turbulence Mach number on viscosity dissipation and pressure dilatation has been considered on the basis of previous work [14], and the correction of the existing compressible $k-\varepsilon$ turbulence model was performed. The equation of turbulent kinetic energy $k$ is expressed as

$$
\begin{aligned}
\frac{\partial(\bar{\rho} k)}{\partial t}+\frac{\partial\left(\bar{\rho} \tilde{u}_{j} k\right)}{\partial x_{j}}= & D_{i j}+P_{i j}+\Pi_{i j} \\
& -\varepsilon_{i j}-\frac{\overline{\rho^{\prime} u_{i}^{\prime \prime}}}{\bar{\rho}} \frac{\partial \bar{p}}{\partial x_{i}}
\end{aligned}
$$

where the detailed expressions of the various terms on the right-hand side are as follows.

Stress Divergence

$$
D_{i j}=-\frac{\partial}{\partial x_{j}}\left[\frac{1}{2} \overline{\rho u_{i}^{\prime \prime} u_{i}^{\prime \prime} u_{j}^{\prime \prime}}+\left(\overline{p^{\prime} u_{i}^{\prime \prime}}\right) \delta_{i j}-\overline{\tau_{i j}^{\prime} u_{i}^{\prime \prime}}\right]
$$

This includes turbulent kinetic energy divergence, $(1 / 2) \rho u_{i}^{\prime \prime} u_{i}^{\prime \prime} u_{j}^{\prime \prime}$, pressure divergence $\left(\overline{p^{\prime} u_{i}^{\prime \prime}}\right) \delta_{i j}$, and viscosity divergence, $-\overline{\tau_{i j}^{\prime} u_{i}^{\prime \prime}}$.

Production of Turbulent Kinetic Energy

$$
P_{i j}=-\overline{\rho u_{i}^{\prime \prime} u_{j}^{\prime \prime}} \frac{\partial \tilde{u}_{i}}{\partial x_{j}}
$$

Pressure Dilatation

$$
\Pi_{i j}=\overline{p^{\prime} \frac{\partial u_{i}^{\prime \prime}}{\partial x_{i}}}
$$

Turbulent Kinetic Energy Dissipation

$$
\varepsilon_{i j}=\bar{\rho} \varepsilon=\overline{\tau_{i j}^{\prime} \frac{\partial u_{i}^{\prime \prime}}{\partial x_{j}}}
$$

By using gradient modeling, we have

$$
\begin{aligned}
& -\frac{1}{2} \overline{\rho u_{i}^{\prime \prime} u_{i}^{\prime \prime} u_{j}^{\prime \prime}}-\overline{p^{\prime} u_{i}^{\prime \prime}} \delta_{i j}= \\
& \frac{\mu_{\mathrm{i}}}{\sigma_{\mathrm{k}}} \frac{\partial k}{\partial x_{j}}-\overline{\rho^{\prime} u_{i}^{\prime \prime}}=\frac{\mu_{i}}{\bar{\rho}} \frac{\partial \bar{\rho}}{\partial x_{i}}
\end{aligned}
$$

From the hypothesis of Boussinesq, we obtain

$$
-\overline{\rho u_{i}^{\prime \prime} u_{j}^{\prime \prime}}=\mu_{\mathrm{t}}\left(\frac{\partial \tilde{u}_{i}}{\partial x_{j}}+\frac{\partial \tilde{u}_{j}}{\partial x_{i}}\right)-\frac{2}{3}\left(\bar{\rho} k+\mu_{\mathrm{t}} \frac{\partial \tilde{u}_{k}}{\partial x_{k}}\right)
$$

The fluctuation dilatation $d^{\prime}=\left(\partial u_{i}^{\prime \prime} / \partial x_{j}\right)$ is included in the pressure dilatation $\Pi_{i j}$ and the turbulent kinetic energy dissipation $\varepsilon_{i j}$. The compressibility of flow is chiefly reflected in the density fluctuation, which is related to the fluctuation dilatation $d^{\prime}$. 


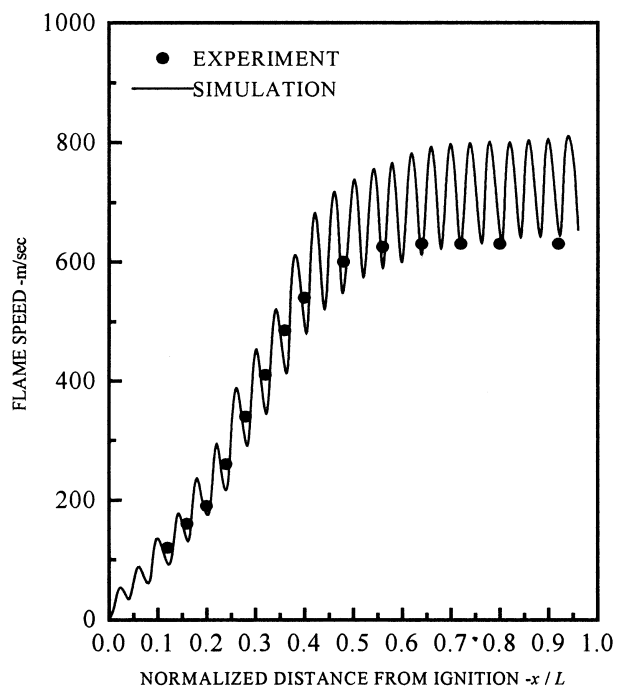

FIG. 10. Variation of centerline speed in an acetylene/ air mixture flame with distance from ignition $(\mathrm{BR}=0.438$, $\Phi=0.58)$.

For high Reynolds number compressible turbulence, in the case of homogeneity, the turbulent kinetic energy dissipation can be expressed as [14]

$$
\bar{\rho} \varepsilon=\mu\left(\overline{\omega_{i} \omega_{i}}+\frac{4}{3} \mu \overline{d^{\prime} d^{\prime}}\right)=\bar{\rho} \varepsilon_{\mathrm{s}}+\bar{\rho} \varepsilon_{\mathrm{d}}
$$

where $\omega_{\mathrm{i}}=\nabla \times u_{i}^{\prime \prime}$ and $d^{\prime}=\nabla \cdot u_{i}^{\prime \prime}, \bar{\rho} \varepsilon_{\mathrm{s}}=\mu \overline{\omega_{\mathrm{i}} \omega_{\mathrm{i}}}$ is the solenoidal dissipation, and $\bar{\rho} \varepsilon_{\mathrm{d}}=(4 / 3) \mu \overline{d^{\prime} d^{\prime}}$ is the compressible dilatation dissipation. According to the discussion of previous works [15,16], a plausible parametric relationship is

$$
\begin{aligned}
\varepsilon_{\mathrm{d}} & =0.15 H\left(M_{\mathrm{t}}\right) \varepsilon_{\mathrm{s}} H\left(M_{\mathrm{t}}\right) \\
& =\left\{\begin{array}{cc}
\left(M_{\mathrm{t}}^{2}-M_{\mathrm{t} 0}^{2}\right) & M_{\mathrm{t}} \geq M_{\mathrm{t} 0} \\
0 & M_{\mathrm{t}}<M_{\mathrm{t} 0}
\end{array}\right.
\end{aligned}
$$

where for the turbulence Mach number $M_{\mathrm{t}}=$ $\sqrt{2 k} / c, c$ is the sound speed, and $M_{\mathrm{t} 0}$ equals 0.25 .

The pressure dilatation $\overline{p^{\prime} d^{\prime}}$ can be derived from the following relationship $[17,18]$ :

$$
\overline{p^{\prime} d^{\prime}}=0.2 M_{\mathrm{t}}^{2} \bar{\rho} \varepsilon_{\mathrm{s}}-0.4 M_{\mathrm{t}}^{2} \bar{\tau}_{\mathrm{ij}} \frac{\partial \tilde{u}_{i}}{\partial x_{j}}
$$

In all the equations above, a prime represents fluctuations with respect to the Reynolds average, while a double prime signifies fluctuations with respect to the Favre average.

The source term related with the rate of combustion is included in the species conservation. The time mean rate of combustion is treated by an eddy break-up model as follows [19].

$$
\bar{R}_{\mathrm{fu}}=-C_{\mathrm{EBU}} \bar{\rho} \frac{\varepsilon}{k} \tilde{Y}_{\mathrm{lim}}
$$

where $\tilde{Y}_{\lim }=\min \left[\tilde{Y}_{\mathrm{f}}, \tilde{Y}_{\mathrm{O}_{2}} / \beta, \tilde{Y}_{\mathrm{p}}\right], C_{\mathrm{EBU}}=4.0$. $\tilde{Y}_{\mathrm{f}}$ is the mass fraction of fuel, $\tilde{Y}_{\mathrm{O}_{2}}$ the mass fraction of oxygen, $\tilde{Y}_{\mathrm{P}}$ that of product, and $\beta$ the coefficient in the mass-based combustion equation: $\{F\}+\beta\left\{\mathrm{O}_{2}\right\} \rightarrow(1+\beta)\{P\}$.

The above nomenclature is defined in the papers cited.

The comparison of predicted and measured flame speeds in an acetylene/air mixture along the centerline of the flame tube is shown in Fig. 10. This figure shows the general feature of flame propagation, which is a wavy increase of speed. The flame accelerates generally due to the turbulence produced by the obstacles, and the alternating acceleration and deceleration of the flame speed results from the periodic variation of the area of the flame tube when the flame passes through the obstacles.

Figure 11 shows a comparison between predictions and experiments in an acetylene/air mixture of two overpressure traces versus time (position $\mathrm{A}$ is near the closed end at $x / L=0.08$, and position $\mathrm{B}$ is near the open end at $x / L=0.92$ ). It is found that the later time history of the overpressure of the simulation does not agree well with the experiment. A possible explanation for this is the fact that the mixture was presumably quiescent before ignition in the simulation, whereas the initial velocities of the mixture were not zero in the experiment, possibly giving rise to a faster overpressure decay. Nevertheless, good agreement in the peak overpressures can be seen in both positions. It is reasonable to use the above turbulent flow model to analyze the development of a premixed flame in the obstructed tube. However, neither predictions of transition to detonation nor of the composition range of flame quenching can be made by the present model in that the effect of the shock wave on combustion and of chemical kinetic effects at quenching are not involved in the model.

\section{Conclusion}

Premixed flame propagation in an obstructed semiopen tube is a complex combustion process in which the combustion and the flow ahead of the flame are intimately coupled. In the present study, an investigation of the dependence of turbulent flame acceleration and overpressure in the tube on the configuration of obstacles has been carried out by changing the obstacle shape, spacing, and blockage ratio.

The influence of the obstruction characteristics on the flame speed and overpressure is different in the various flame regimes. In the low combustion velocity regime, the highest terminal flame speed is obtained with the blockage ratio of $\mathrm{BR}=0.3-0.4$. In the choking regime, the maximum flame speed is insensitive to the blockage ratio, while a blockage 


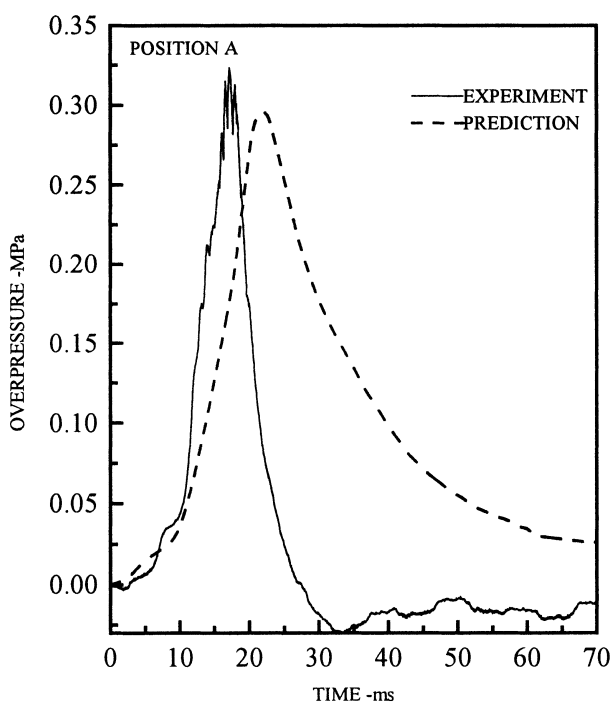

Fig.11-a) The position A $\quad(x / L=0.08)$

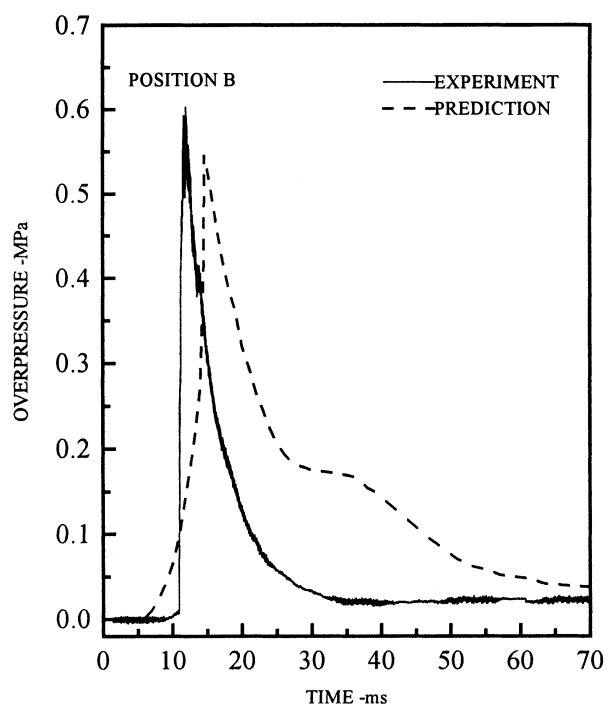

Fig.11-b) The position B $(x / L=0.92)$

FIG. 11. Variation of overpressure in acetylene/air mixtures with time for two positions along the tube $(\mathrm{BR}=$ $0.438, \Phi=0.58$ ). ratio of about 0.5 generates the highest peak overpressure. In the detonation regime, the maximum flame speed and overpressure decrease with increasing blockage ratio due to the severe momentum losses induced by the obstacles, and at the same time, the detonative range is observed to become narrower.

With the correction of viscosity dissipation and pressure dilatation, the modified $k-\varepsilon$ two-dimension turbulent model and EBU combustion model have been used to predict the flame acceleration and the development of overpressure in the tube in the deflagration regime. The comparison of calculated overpressure and flame speeds with experimental data shows good agreement.

\section{Acknowledgments}

This work was supported by the National Key Fundamental Research and Development Program of China under grant G1999022305.

\section{REFERENCES}

1. Moen, I. O., Donato, M., Knystautas, R., and Lee, J. H., Combust. Flame 39:21 (1980).

2. Chan, C. K., and Greig, D. R., Proc. Combust. Inst. 22:1733 (1988).

3. Teodorczyk, A., Lee, J. H. S. and Knystautas, R., Proc. Combust. Inst. 23:735 (1990).

4. Lee, J. H. S., Knystautas, R., and Freiman, A., Combust. Flame 56:227 (1984).

5. Lee, J. H., Knystautas, R., and Chan, C. K., Proc. Combust. Inst. 20:1663 (1984).

6. Peraldi, O., Knystautas, R., and Lee, J. H., Proc. Combust. Inst. 21:1629 (1986).

7. Moen, I. O., Lee, J. H. S., Hjertager, B. H., Fuhre, K., and Eckhoff, R. K., Combust. Flame 47:31 (1982).

8. Hjertager, B. H., Fuhre, K., Parker, S. J., and Bakke, J. R., Prog. Astronaut. Aeronaut. 94:504 (1984).

9. Chan, C., Moen, I. O., and Lee, J. H. S., Combust. Flame 49:27 (1983).

10. Bradley, D., and Mitcheson, A., Combust. Flame 32:221 (1978).

11. Bradley, D., and Mitcheson, A., Combust. Flame 32:237 (1978).

12. Yu, L. X., Sun, W. C., and Wu, C. K., in Chinese, J. Combust. Sci. Technol. 7(3):223 (2001).

13. Craig, B. G., Proc. Combust. Inst. 10:863 (1964).

14. Sarkar, S., Erlebacher, G., Hvssaini, M. Y., and Kreiss, H. O., J. Fluid Mech. 227:473 (1991).

15. Wilcox, D. C., AIAA paper 91-1785.

16. Zeman, O., Phys. Fluids A 2(2):178 (1990).

17. Sarkar, S., Phys. Fluids A 4(12):2674 (1992).

18. Liang, D. W., and Wang, G. Q., in Chinese Acta Aerodynamic Sinica 18(1):98 (2000).

19. Spalding, D. B., Combust. Sci. Technol. 13:3 (1976). 